\title{
Model of vector leptoquarks in view of the $B$-physics anomalies
}

\author{
Lorenzo Calibbi ${ }^{*}$ \\ CAS Key Laboratory of Theoretical Physics, Institute of Theoretical Physics, \\ Chinese Academy of Sciences, Beijing 100190, China \\ Andreas Crivellin ${ }^{\dagger}$ \\ Paul Scherrer Institut, CH-5232 Villigen PSI, Switzerland \\ Tianjun $\mathrm{Li}^{\ddagger}$ \\ CAS Key Laboratory of Theoretical Physics, Institute of Theoretical Physics, \\ Chinese Academy of Sciences, Beijing 100190, China \\ and School of Physical Sciences, University of Chinese Academy of Sciences, \\ No. 19A Yuquan Road, Beijing 100049, China
}

(Received 6 September 2017; published 4 December 2018)

\begin{abstract}
Lepton number as a fourth color is an intriguing theoretical idea which is combined with a possible leftright symmetry within the famous Pati-Salam (PS) model. In the conventional PS model, a spontaneous breaking of the PS gauge group down to the SM, one can only take place at very high scales (above the PeV scale) due to the stringent bounds from $K_{L} \rightarrow \mu e$ and $K \rightarrow \pi \mu e$ induced by the resulting vector leptoquarks. In this paper, we show that these constraints can be avoided once additional vectorlike fermions are introduced and, thus, a breaking at the $\mathrm{TeV}$ scale is possible. We consider the flavor phenomenology of this model in the context of the intriguing hints for new physics in semileptonic $B$ decays. The necessary violation of lepton flavor universality is induced by mixing SM and vectorlike fermions. Concerning $R(D)$ and $R\left(D^{*}\right)$, we find that sizable effects are possible while respecting the bounds from other flavor observables but predicting a large enhancement of $B_{s} \rightarrow \tau^{+} \tau^{-}$. Furthermore, also in $b \rightarrow s \ell^{+} \ell^{-}$transitions, the observed deviations from the SM predictions [including $R(K)$ and $R\left(K^{*}\right)$ ] can be explained with natural values for the free parameters of the model without any fine-tuning, predicting sizable decay rates for $b \rightarrow s \tau \mu$. Finally, the anomaly in the anomalous magnetic moment of the muon can be accounted for by a loop contribution involving the vector leptoquark and vectorlike leptons.
\end{abstract}

DOI: 10.1103/PhysRevD.98.115002

\section{INTRODUCTION}

Until now, the Large Hadron Collider at CERN did not directly observe any particles beyond the ones present in the standard model (SM) of particle physics. However, we have accumulated intriguing hints for lepton flavor universality (LFU) violation in semileptonic $B$ decays within recent years. Most prominently, there exist deviations from the SM predictions in $b \rightarrow s \mu^{+} \mu^{-}$above the $5 \sigma$ level $[1]^{1}$ and the

\footnotetext{
*calibbi@itp.ac.cn

andreas.crivellin@cern.ch

\#tli@itp.ac.cn

${ }^{1}$ Including only $R(K)$ and $R\left(K^{*}\right)$ in the fit the significance is at the $4 \sigma$ level [2-8].

Published by the American Physical Society under the terms of the Creative Commons Attribution 4.0 International license. Further distribution of this work must maintain attribution to the author(s) and the published article's title, journal citation, and DOI. Funded by SCOAP.
}

combination of the ratios $R(D)$ and $R\left(D^{*}\right)$ differs by $4.1 \sigma$ from its SM prediction [9]. While $R(D)$ and $R\left(D^{*}\right)$ measure LFU violation in the charged current $B$ decays of the type $b \rightarrow c \ell \nu$, the fit to the $b \rightarrow s \ell^{+} \ell^{-}$data also suggests the violation of LFU due to the measurement of $R(K)$ [10] and $R\left(K^{*}\right)$ [11]. This implies a possible connection between these two classes of anomalies and motivates the search for a simultaneous explanation [12-28].

The vector leptoquark $S U(2)$ singlet with hypercharge $-4 / 3$ is a natural candidate for a simultaneous explanation of $R(D)$ and $R\left(D^{*}\right)$ together with $b \rightarrow s \ell^{+} \ell^{-}$data. First of all, it automatically fulfills the requirement that downquarks do not couple at tree level to neutrinos and, therefore, avoids the stringent bounds from $B \rightarrow K^{(*)} \nu \nu$. This allows for large flavor violating couplings to quarks such that one can get a sizable effect in $R(D)$ and $R\left(D^{*}\right)$ with $\mathrm{TeV}$ scale masses such that the bounds from direct searches [29] as well as from electroweak (EW) precision data [30] can be avoided [25]. In addition, unlike models with charged Higgses [31-36], the vector leptoquark leaves 
the $q^{2}$ distribution in $R\left(D^{(*)}\right)$ invariant (which is in good agreement with data [37-39]) and does not lead to an huge enhancement of $B_{c} \rightarrow \tau \nu$ which is incompatible with experiments [38,40-42]. Finally, it gives a $C_{9}=-C_{10}$-like effect in $b \rightarrow s \ell^{+} \ell^{-}$transitions and, therefore, gives a good fit to data. However, a compelling renormalizable model giving rise to this leptoquark is still missing.

Interestingly, the vector leptoquark singlet with hypercharge $-4 / 3$ is contained within the theoretically very appealing PS model as a $S U(4)$ gauge boson. However, in the conventional model, the bounds on the symmetry breaking scale from $K_{L} \rightarrow \mu e$ and $K \rightarrow \pi \mu e$ are so strong (at the PeV scale) $[43,44]$ that any other observable effects in flavor physics are ruled out from the outset. Therefore, it must be extended if one aims at a realization at the $\mathrm{TeV}$ scale. In this article, we will construct a model based on the Pati-Salam gauge group in which the bounds from $K_{L} \rightarrow$ $\mu e$ and $K \rightarrow \pi \mu e$ can be avoided. Furthermore, another crucial feature of the PS leptoquarks is that it allows for a low-energy realization since it does not lead to proton decay at any loop level.

For this purpose, we add to the original PS model three pairs of fermions in the fundamental representation of $S U$ (4) with vectorlike mass terms. These fermions can be considered as heavy vectorlike generations. The mixing between them and the light SM particles is in general flavor dependent. Therefore, the model can have interesting effects in flavor physics, in particular, it could explain the hints for new physics in $b \rightarrow s \ell^{+} \ell^{-}, R\left(D^{(*)}\right)$ and also the measurement of the anomalous magnetic moment $(\mathrm{AMM})$ of the muon.

\section{THE MODEL}

Our starting point is the PS model [45] with the gauge group $S U(4) \times S U(2)_{L} \times S U(2)_{R}$. Thus, left-handed fermions are $S U(2)_{L}$ doublets and right-handed fermions form $S U(2)_{R}$ doublets. This necessarily leads to the introduction of three right-handed neutrinos. In our model, we extend the fermion content of the original model having now 6 fermion fields $X_{i}^{L, R}, Y_{i}^{L, R}, Z_{i}^{L, R}$ as well as (at least) two more Higgs field $\Sigma^{1,2}$. These fields transform under the PS gauge group and one additional Peccei-Quinn-like $U(1)$ group as shown in Table I.

Here, the superscripts $L$ and $R$ label the chirality of the fields and $i=1,2,3$ is a flavor index. In the absence of the fields $Y$ and $Z$, the fields $X$ would be chiral fermions resembling the SM fermions.

In the following, we will not explicitly specify the EW symmetry breaking sector whose Higgs fields are, therefore, not included in Table I. However, we know that due to the decoupling theorem, the symmetry breaking sector must reduce, in the limit of heavy additional Higgses, to one light $S U(2)$ doublet with vev $v$ giving rise the chiral fermion and weak gauge boson masses.
TABLE I. Field content of the model. Alternatively, one could use instead of $\Sigma_{1,2}^{X, Y}$ two fields $\Sigma_{i j}^{X}$, which transforms as $\overline{3} \otimes 3$ under a possible flavor symmetry.

\begin{tabular}{ccccc}
\hline \hline & $S U(4)$ & $S U(2)_{L}$ & $S U(2)_{R}$ & $U(1)_{P Q}$ \\
\hline$X_{i}^{L}$ & 4 & 2 & 1 & 0 \\
$Y_{i}^{L}$ & 4 & 2 & 1 & -1 \\
$Y_{i}^{R}$ & 4 & 2 & 1 & 1 \\
$X_{i}^{R}$ & 4 & 1 & 2 & 0 \\
$Z_{i}^{R}$ & 4 & 1 & 2 & -1 \\
$Z_{i}^{L}$ & 4 & 1 & 2 & 1 \\
$\Sigma_{1}^{X}$ & $\overline{4} \otimes 4$ & 1 & 1 & -1 \\
$\Sigma_{2}^{X}$ & $\overline{4} \otimes 4$ & 1 & 1 & -1 \\
$\Sigma_{1}^{Y}$ & $\overline{4} \otimes 4$ & 1 & 1 & -2 \\
$\Sigma_{2}^{Y}$ & $\overline{4} \otimes 4$ & 1 & 1 & -2 \\
\hline \hline
\end{tabular}

A possible completion of the above-sketched model, including the EW-breaking sector, will be given in Sec. V. In our phenomenological discussion, we are not considering the implications of the extended Higgs sector, but rather we only include the pseudo-Goldstone bosons by working in unitary gauge. This approach is model independent in the sense that including additional physical Higgses would imply focusing on a specific UV realization of the model.

\section{A. Fermion masses}

Let us consider for simplicity only the $S U(2)_{L}$ doublet fermions $\left(X_{i}^{L}, Y_{i}^{L, R}\right)$. The corresponding results for the $S U(2)_{R}$ follow in a straightforward way and they are not necessary for explaining the flavor anomalies as we will see later. Therefore, we can write down the following mass terms after the new scalar fields $\Sigma_{1,2}^{X, Y}$ acquire their vevs $v_{\Sigma_{1,2}^{X, Y}}$

$$
\begin{aligned}
-\mathcal{L} \supset & v_{\Sigma_{1}^{X}}^{a b} x_{i j} \bar{X}_{i}^{a L} Y_{j}^{b R}+v_{\Sigma_{1}^{Y}}^{a b} y_{i j} \bar{Y}_{i}^{a L} Y_{j}^{b R} \\
& +v_{\Sigma_{2}^{X}}^{a b} x_{i j}^{\prime} \bar{X}_{i}^{a L} Y_{j}^{b R}+v_{\Sigma_{2}^{Y}}^{a b} y_{i j}^{\prime} \bar{Y}_{i}^{a L} Y_{j}^{b R}+\text { H.c. }
\end{aligned}
$$

Here $a$ and $b$ are $S U(4)$ indices, and we denoted the Yukawalike couplings by $x_{i j}^{(\prime)}$ and $y_{i j}^{(\prime)}$. Note that our assignment for the PQ charges was choosen in such a way that it avoids bare mass terms for the fermions before PS symmetry breaking. Therefore, the masses of the vectorlike fermions are, for perturbative couplings, at most of the order of the $S U(4)$ breaking scale, which we assume to be around the $\mathrm{TeV}$ scale. After $\Sigma_{1,2}^{X, Y}$ acquire their vevs $S U(4)$ is broken down to $S U(3)_{C} \times U(1)_{B-L}$ and quarks and leptons become distinguishable. Decomposing the $S U(4)$ multiplets as

$$
Y_{R}=\left(\begin{array}{c}
Q_{R}^{\prime} \\
L_{R}^{\prime}
\end{array}\right)_{i}, \quad Y_{L}=\left(\begin{array}{c}
Q_{L} \\
\ell_{L}
\end{array}\right)_{i}, \quad X_{L}=\left(\begin{array}{c}
q_{L} \\
L_{L}
\end{array}\right)_{i}
$$


we see that $Q$ and $q$ are $S U(3)_{c}$ triplets corresponding to quarks, while $\ell$ and $L$ are $S U(3)_{c}$ singlets and, thus, correspond to leptons. Expanding Eq. (1) into components, we find

$$
\mathcal{L} \supset-\left(m_{i j}^{Q} \bar{q}_{i L}+M_{i j}^{Q} \bar{Q}_{i L}\right) Q_{j R}^{\prime}-\left(M_{i j}^{L} \bar{L}_{i L}+m_{i j}^{L} \bar{\ell}_{i L}\right) L_{j R}^{\prime},
$$

with

$$
\begin{array}{ll}
m_{i j}^{Q}=v_{\Sigma_{1}^{X}}^{11} x_{i j}+v_{\Sigma_{2}^{X}}^{11} x_{i j}^{\prime}, & m_{i j}^{L}=v_{\Sigma_{1}^{Y}}^{22} y_{i j}+v_{\Sigma_{2}^{Y}}^{22} y_{i j}^{\prime} \\
M_{i j}^{L}=v_{\Sigma_{1}^{X}}^{22} x_{i j}+v_{\Sigma_{2}^{X}}^{22} x_{i j}^{\prime}, & M_{i j}^{Q}=v_{\Sigma_{1}^{Y}}^{11} y_{i j}+v_{\Sigma_{2}^{Y}}^{11} y_{i j}^{\prime}
\end{array}
$$

Here the superscript 11 corresponds to a $3 \times 3$ unit matrix in color space while 22 represents only a single number. Here $v_{\Sigma_{1,2}^{X, Y}}^{12}=v_{\Sigma_{1,2}^{X, Y}}^{21}=0$ such that $S U(3)_{c}$ remains unbroken. We further assume $v_{\Sigma_{1}^{X, Y}}^{11} \gg v_{\Sigma_{2}^{X, Y}}^{11}$ and $v_{\Sigma_{1}^{X, Y}}^{22} \ll v_{\Sigma_{2}^{X, Y}}^{22}$, such that the mass terms are dominantly given by

$$
\begin{aligned}
m_{i j}^{Q} \simeq v_{\Sigma_{1}^{X}}^{11} x_{i j}, & m_{i j}^{L} \simeq v_{\Sigma_{2}^{Y}}^{22} y_{i j}^{\prime} \\
M_{i j}^{L} \simeq v_{\Sigma_{2}^{X}}^{22} x_{i j}^{\prime}, & M_{i j}^{Q} \simeq v_{\Sigma_{1}^{Y}}^{11} y_{i j}
\end{aligned}
$$

Therefore, $M_{i j}^{L, Q}$ are the vectorlike mass terms while $m_{i j}^{L, Q}$ provides the mixing of the vectorlike fermions with the light (SM) ones. The study of the corresponding scalar potential is not trivial and requires future studies.

Without loss of generality, one can choose $M^{Q}$ and $M^{L}$ to be diagonal in flavor space. In addition, we assume that $m^{Q, L}$ is diagonal in the same basis and for simplicity (without affecting the final results) that $M^{Q, L}$ is proportional to the unit matrix:

$$
\begin{aligned}
M_{i j}^{Q, L} & =M^{Q, L} \delta_{i j}, \\
m_{i j}^{Q, L} & =\left(\begin{array}{ccc}
m_{1}^{Q, L} & 0 & 0 \\
0 & m_{2}^{Q, L} & 0 \\
0 & 0 & m_{3}^{Q, L}
\end{array}\right)_{i j} .
\end{aligned}
$$

While the structure above is certainly not generic, it can be the consequence of an underlying flavor symmetry. In fact, if $Q_{L}$ and $Q_{R}^{\prime}\left(Q_{L}\right.$ and $\left.L_{R}^{\prime}\right)$ are triplets of $S U(3), M^{Q}\left(M^{L}\right)$ does not break the symmetry and is, thus, proportional to the unit matrix. If on the contrary, $q_{L}$ and $\ell_{L}$ are antitriplets, $m^{Q, L}$ are generated by $S U(3)$-breaking terms $\phi \phi / \Lambda$, where $\phi$ collectively denote the $S U(3)$-triplet scalar fields (flavons) whose vevs break $S U(3)$ and $\Lambda$ a cutoff scale. The texture of $m^{Q, L}$ then follows from the flavor directions of the flavons' vevs. As an example, one can introduce two flavons, $\phi_{3}$ and $\phi_{2}$, with $\left\langle\phi_{3}\right\rangle=\left(0,0, v_{3}\right),\left\langle\phi_{2}\right\rangle=\left(0, v_{2}, 0\right)$. Distinguishing these two fields by an additional parity, the texture in Eq. (6) is obtained with $m_{3}^{Q, L} \sim v_{3}^{2} / \Lambda, m_{2}^{Q, L} \sim v_{2}^{2} / \Lambda$,
$m_{1}^{Q, L} \sim 0$. Therefore, the mixing with electrons is absent, and thus the vector LQs will not couple to them. ${ }^{2}$

Given the structure in Eq. (6), the mass matrices for quarks and leptons decompose each into three (one for each generation) rank one matrices diagonalized by the rotations

$$
\begin{aligned}
\left(\begin{array}{l}
q_{i L} \\
Q_{i L}
\end{array}\right) & \rightarrow\left(\begin{array}{cc}
c_{i Q} & -s_{i Q} \\
s_{i Q} & c_{i Q}
\end{array}\right)\left(\begin{array}{l}
q_{i L} \\
Q_{i L}
\end{array}\right) \\
\left(\begin{array}{l}
\ell_{i L} \\
L_{i L}
\end{array}\right) & \rightarrow\left(\begin{array}{cc}
c_{i L} & -s_{i L} \\
s_{i L} & c_{i L}
\end{array}\right)\left(\begin{array}{l}
\ell_{i L} \\
L_{i L}
\end{array}\right) .
\end{aligned}
$$

As stated above, we do not explicitly specify the UV completion of the Higgs sector responsible for the EW symmetry breaking but rather use the decoupling theorem asserting that there is one light $S U(2)_{L}$ doublet with vev $v$ giving rise to the chiral fermion and weak gauge boson masses. We can now write down the usual Yukawa couplings and diagonalize the resulting $3 \times 3$ matrices using biunitary transformations

$$
q_{i L} \rightarrow U_{i j}^{q L} q_{j L}, \quad \ell_{i L} \rightarrow U_{i j}^{\ell L} \ell_{j L},
$$

with $q=u, d$ and the corresponding expression for righthanded fields. For our final results, only the misalignment between left-handed quark and leptons

$$
U_{f i}^{q \ell L}=U_{j f}^{q L *} U_{j i}^{\ell L},
$$

as well as the CKM matrix $V_{f i}^{\mathrm{CKM}}=U_{j f}^{u L *} U_{j i}^{d L}$ are important. Note that in the following, we work in the down basis, i.e., CKM rotations are only present once left-handed upquarks are involved. We neglect Higgs couplings involving chiral and vectorlike fermions in our phenomenological analysis.

In analogy to the $S U(2)_{L}$ sector, we embedded the fermions charged under $S U(2)_{R}$ in the following representations:

$$
Z_{L}=\left(\begin{array}{c}
Q_{L}^{\prime} \\
L_{L}^{\prime}
\end{array}\right)_{i}, \quad Z_{R}=\left(\begin{array}{c}
Q_{R} \\
\ell_{R}
\end{array}\right)_{i}, \quad X_{R}=\left(\begin{array}{c}
q_{R} \\
L_{R}
\end{array}\right)_{i}
$$

and the above discussion about masses and mixing can be replicated for the RH fermions of the SM.

\section{B. Couplings of fermions to gauge bosons}

After breaking of the $S U(4)$ symmetry, its 15 generators correspond to 8 massless gluons, 6 leptoquarks $\left(V^{\mu}+\bar{V}^{\mu}\right)$,

\footnotetext{
${ }^{2}$ The absence of couplings to the electron at tree-level could alternatively be assured by an Abelian flavor symmetry under which all fermions are equally charged except the electron. Furthermore, even though electron couplings will be generated at the loop level [46], the absence of $\mu e$ couplings is RGE invariant and, therefore, no effect in $\mu \rightarrow e \gamma$ [47] or $K \rightarrow \mu e$ is generated.
} 


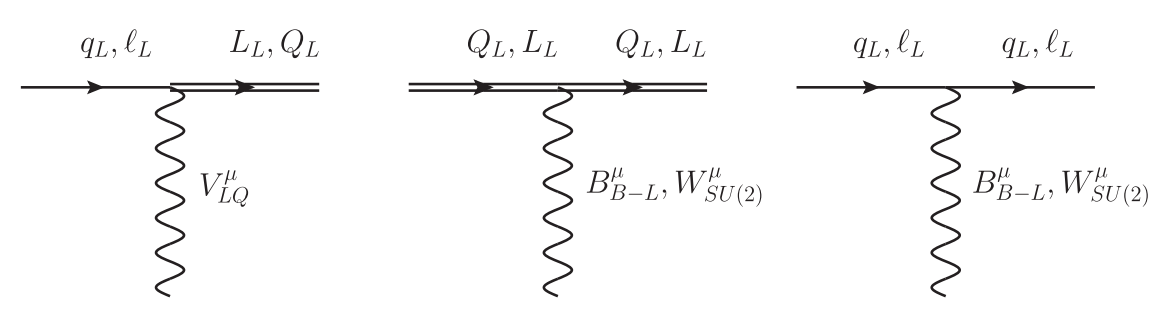

FIG. 1. Couplings of the gauge bosons to the heavy vectorlike fermions $(L, Q)$ and light SM-like fermions $(\ell, q)$. After mixing among SM-like and vectorlike fermions, the couplings to the leptoquark result become flavor nonuniversal, whereas the couplings to the other gauge bosons (in particular the one associated to $B-L$ ) remain flavor diagonal.

and one $B-L$ gauge boson $\left(Z^{\prime \mu}\right)$. As we can see from Fig. 1, after mixing of $q(\ell)$ with $Q(L)$ the couplings of the $B-L$ gauge boson remains flavor universal with strength $\sqrt{3 / 8} g_{s} / 3$ for quarks, $\sqrt{3 / 8} g_{s}$ for leptons. The flavor universality of the $Z^{\prime}$ coupling is the result of the unitarity of the mixing matrices. Therefore, we did not need to assume any alignment between the vectorlike mass terms and the Yukawa couplings to obtain this desirable feature.

Since we do not completely specify the Higgs sector (a possible realization is given in Sec. V), we take the masses of the $B-L$ gauge boson and the leptoqaurks as free parameters. However, the masses should be of the same order, but due to the strong constraints from $Z^{\prime}$ searches, ${ }^{3}$ we assume that the $B-L$ gauge boson is heavier than the leptoquarks. Such a mass splitting can be achieved if the PS symmetry breaking is due to the vev of a scalar in the symmetric representation of $S U(4)$. In fact, if the dominant contribution to the gauge boson masses is given by the field $\Phi$ of Table II, which is in the $(10,1,3)$ representation of the PS group, one obtains $M_{L Q}^{2}=g_{4}^{2} v_{\Phi}^{2}$ and $M_{Z^{\prime}}^{2}=$ $3 g_{4}^{2} v_{\Phi}^{2}+2 g_{R}^{2} v_{\Phi}^{2}$, which gives $M_{Z^{\prime}} \approx 2 \times M_{L Q}$. Furthermore, the limit on the $Z^{\prime}$ mass can be significantly weakened by introducing additional fermions which are charged under $B-L$ only, such as extra sterile neutrinos. This does not only decrease the branching ratio to muons and electrons (the modes which give the strongest bound [48]) but also increases the total width, making the detection more difficult [29]. Finally, as we will see later, if we only aimed at a smaller effect in $b \rightarrow c \tau \nu$ processes rather than accounting for the central value, the gauge bosons (including the $Z^{\prime}$ ) can be heavier.

Let us now consider the couplings of the vectorleptoquark $V^{\mu}$. Here, the rotations in Eq. (7) induced by the mixing between vectorlike and SM fermions do not drop out, as it is apparent from Fig. 1. In addition, after EW symmetry breaking, the misalignment between the rotations needed to diagonalize the light quark and lepton mass matrices $U_{f i}^{q \ell L}$, cf. Eq. (9), enters in the coupling of $V^{\mu}$ with the SM fermion doublets:

$$
\begin{aligned}
\mathcal{L} \supset & -\frac{g_{s}}{\sqrt{2}} U_{f i}^{q \ell L}\left(\begin{array}{c}
\bar{q}_{f}^{L} \\
\bar{Q}_{f}^{L}
\end{array}\right) \gamma_{a}^{\mu} P_{L}\left(\begin{array}{c}
\ell_{i}^{L} \\
L_{i}^{L}
\end{array}\right)_{b} \\
& \times\left(\begin{array}{cc}
c_{i}^{Q} s_{i}^{L}+c_{i}^{L} s_{i}^{Q} & c_{i}^{L} c_{i}^{Q}-s_{i}^{L} s_{i}^{Q} \\
c_{i}^{L} c_{i}^{Q}-s_{i}^{L} s_{i}^{Q} & -c_{i}^{Q} s_{i}^{L}-c_{i}^{L} s_{i}^{Q}
\end{array}\right)_{a b} V_{\mu}+\text { H.c. }
\end{aligned}
$$

Considering for illustration only the second and third generations, which are of interest for our phenomenological study, we find

$$
\mathcal{L} \supset \kappa_{i j} \bar{q}_{i}^{L} \gamma^{\mu} P_{L} \ell_{j}^{L} V_{\mu}+\text { H.c. } \quad \text { with } \quad \kappa_{i j}=\frac{-g_{s}}{\sqrt{2}}\left(\begin{array}{ccc}
c_{1}^{Q} s_{1}^{L}+c_{1}^{L} s_{1}^{Q} & 0 & 0 \\
0 & \left(c_{2}^{Q} s_{2}^{L}+c_{2}^{L} s_{2}^{Q}\right) c_{23}^{q \ell} & -s_{23}^{q \ell}\left(c_{2}^{Q} s_{2}^{L}+c_{2}^{L} s_{2}^{Q}\right) \\
0 & \left(c_{2}^{Q} s_{2}^{L}+c_{2}^{L} s_{2}^{Q}\right) s_{23}^{q \ell} & c_{23}^{q \ell}\left(c_{3}^{Q} s_{3}^{L}+c_{3}^{L} s_{3}^{Q}\right)
\end{array}\right) \text {. }
$$

Here $s_{23}^{q \ell}, c_{23}^{q \ell}$ are the rotations induced by the misalignment between the SM Yukawa couplings of quarks and leptons,

\footnotetext{
${ }^{3}$ With $g_{B-L}=\sqrt{3 / 8} g_{s} \approx 0.6$ at the TeV scale, we find that a recent ATLAS search for $Z^{\prime} \rightarrow \ell^{+} \ell^{-}$[48] gives (in the narrow width approximation) $M_{Z^{\prime}} \gtrsim 5 \mathrm{TeV}$ if the $Z^{\prime}$ only decays into SM fermions. This is reduced to $M_{Z^{\prime}} \gtrsim 4.6 \mathrm{TeV}$ in the more realistic case in which all decay channels of our $B-L Z^{\prime}$ into the vectorlike fermions are open via a decrease of the $Z^{\prime} \rightarrow \ell^{+} \ell^{-}$ branching ratio by a factor $1 / 3$ and an increase of the total width up to $\approx 0.18 \times M_{Z^{\prime}}$.
}

encoded in Eq. (9). Recall that we assumed that the first generation quarks (in the interaction basis) and leptons do not mix with their SM partners. In this way, effects in $b \rightarrow$ $d \ell^{+} \ell^{-}$or Kaon decays [49] are suppressed and $\mu e$ lepton flavor violation is absent. This ensures that our model is consistent with the bounds from $K_{L} \rightarrow \mu e$ and $K \rightarrow \pi \mu e$ for $\mathrm{TeV}$ scale masses. The suppression of the couplings of the vector leptoquark to first generation fermions can be accounted for by a flavor structure of the mixing mass terms $m^{Q, L}$ resembling the strong hierarchy of the SM 
TABLE II. Possible extension of the field content of the model presented in Sec. II accounting for EW breaking.

\begin{tabular}{lccccc}
\hline \hline & $S U(4)$ & $S U(2)_{L}$ & $S U(2)_{R}$ & $U(1)_{Q}$ & $U(1)_{L}$ \\
\hline$X_{i}^{L}$ & 4 & 2 & 1 & 0 & 1 \\
$Y_{i}^{L}$ & 4 & 2 & 1 & 1 & 0 \\
$Y_{i}^{R}$ & 4 & 2 & 1 & 0 & 0 \\
$X_{i}^{R}$ & 4 & 1 & 2 & 0 & 1 \\
$Z_{i}^{R}$ & 4 & 1 & 2 & 1 & 0 \\
$Z_{i}^{L}$ & 4 & 1 & 2 & 0 & 0 \\
$\Sigma_{L_{1}}$ & $\overline{4} \otimes 4$ & 1 & 1 & 0 & -1 \\
$\Sigma_{L_{2}}$ & $\overline{4} \otimes 4$ & 1 & 1 & 0 & -1 \\
$\Sigma_{Q_{1}}$ & $\overline{4} \otimes 4$ & 1 & 1 & -1 & 0 \\
$\Sigma_{Q_{2}}$ & $\overline{4} \otimes 4$ & 1 & 1 & -1 & 0 \\
$\Phi_{q}$ & 1 & 2 & 2 & 0 & -2 \\
$\Phi_{\ell}$ & 1 & 2 & 2 & -2 & 0 \\
$\Phi$ & 10 & 1 & 3 & -2 & 0 \\
\hline \hline
\end{tabular}

Yukawas, which can be enforced by an underlying flavor symmetry, as in the $S U(3)$ example discussed below Eq. (6). In such a case, mixing with the first generation can be strongly suppressed by simply not introducing a flavon with a vev in the flavor direction $i=1$.

Similarly, couplings of $V^{\mu}$ to right-handed leptons and quarks might arise as an effect of the mixing with the vectorlike fermions in the $S U(2)_{R}$ sector, i.e., the field embedding in Eq. (10). Such couplings should be small (but not necessarily zero) due to the observed patterns in $R\left(D^{(*)}\right)$ and $b \rightarrow s \mu^{+} \mu^{-}$transitions. In our setup, this can be easily achieved by a mild suppression of the SM-like/ vectorlike fermion mixing in the $\mathrm{RH}$ sector.

\section{OBSERVABLES}

\section{A. $R(D)$ and $R\left(D^{*}\right)$}

We define the effective Hamiltonian for $b \rightarrow c \ell v$ transitions as

$$
H_{\mathrm{eff}}^{\ell_{f} \nu_{i}}=\frac{4 G_{F}}{\sqrt{2}} V_{c b} C_{L}^{f i}\left[\bar{c} \gamma^{\mu} P_{L} b\right]\left[\bar{\ell}_{f} \gamma_{\mu} P_{L} \nu_{i}\right]
$$

where in the $\mathrm{SM} C_{L}^{f i}=\delta_{f i}$ and the contribution of our vector leptoquark is given by

$$
C_{L}=\frac{\sqrt{2}}{4 G_{F} V_{c b}} \frac{\kappa_{33}^{*} V_{2 j} \kappa_{j 3}}{M^{2}},
$$

leading to

$$
R\left(D^{(*)}\right) / R\left(D^{(*)}\right)_{\mathrm{SM}}=\left|1+C_{L}\right|^{2},
$$

where we neglected contributions with muon or electron neutrinos. This has to be compared to the experimental measurements of $R\left(D^{*}\right)_{\mathrm{EXP}}=0.304 \pm 0.013 \pm 0.007$ and
$R(D)_{\mathrm{EXP}}=0.407 \pm 0.039 \pm 0.024$, and the corresponding SM predictions, $R\left(D^{*}\right)_{\mathrm{SM}}=0.252 \pm 0.003$ and $R(D)_{\mathrm{SM}}=$ $0.300 \pm 0.008[50,51]$.

\section{B. $b \rightarrow s \ell^{+} \ell^{-}$transitions}

Using the effective Hamiltonian

$$
\begin{aligned}
H_{\mathrm{eff}}^{\ell_{f} \ell_{i}} & =-\frac{4 G_{F}}{\sqrt{2}} V_{t b} V_{t s}^{*} \sum_{a=9,10} C_{a}^{f i} O_{a}^{f i}, \\
O_{9(10)}^{f i} & =\frac{\alpha}{4 \pi}\left[\bar{s} \gamma^{\mu} P_{L} b\right]\left[\bar{\ell}_{f} \gamma_{\mu}\left(\gamma^{5}\right) \ell_{i}\right],
\end{aligned}
$$

we have

$$
C_{9}^{f i}=-C_{10}^{f i}=\frac{-\sqrt{2}}{2 G_{F} V_{t b} V_{t s}^{*}} \frac{\pi}{\alpha} \frac{\kappa_{2 i} \kappa_{3 f}^{*}}{M^{2}} .
$$

The allowed range is given by [1]

$$
-0.36(-0.48) \geq C_{9}^{22}=-C_{10}^{22} \geq(-0.73)-0.87,
$$

at the 2(1) $\sigma$ level. In the case of lepton flavor violating $B$ decays, we use the results of Ref. [52] for the analysis of $B \rightarrow K^{(*)} \tau \mu$ which currently gives the best experimental limits for $\mu \tau$ final states of [53]

$$
\operatorname{Br}[B \rightarrow K \tau \mu]_{\mathrm{EXP}} \leq 4.8 \times 10^{-5},
$$

at $90 \%$ confidence level. For our case of $C_{9}=-C_{10}$, we get

$$
\operatorname{Br}[B \rightarrow K \tau \mu]=1.96 \times 10^{-8}\left(\left|C_{9}^{23}\right|^{2}+\left|C_{9}^{32}\right|^{2}\right) .
$$

Finally, we also get an effect in $B_{s} \rightarrow \tau^{+} \tau^{-}$of

$$
\operatorname{Br}\left(B_{s} \rightarrow \tau^{+} \tau^{-}\right)=\operatorname{Br}\left(B_{s} \rightarrow \tau^{+} \tau^{-}\right)_{\mathrm{SM}}\left(1+\frac{C_{10}^{33}}{C_{10}^{\mathrm{SM}}}\right)^{2},
$$

with $\operatorname{Br}\left(B_{s} \rightarrow \tau^{+} \tau^{-}\right)_{\mathrm{SM}}=(7.73 \pm 0.49) \times 10^{-7} \quad[54,55]$ and $C_{10}^{\mathrm{SM}} \approx-4.3[56,57]$. The current experimental limit is $\operatorname{Br}\left(B_{s} \rightarrow \tau^{+} \tau^{-}\right)_{\mathrm{EXP}} \leq 6.8 \times 10^{-3}[58]$.

\section{C. $\boldsymbol{B}_{s}-\overline{\boldsymbol{B}}_{s}$ mixing}

With $H=C_{1} \bar{s} \gamma^{\mu} P_{L} b \bar{s} \gamma_{\mu} P_{L} b$ we get

$$
C_{1}=-\frac{\kappa_{2 s} \kappa_{3 s}^{*} \kappa_{2 t} \kappa_{3 t}^{*}}{16 \pi^{2}}\left(\frac{D_{6}}{4 M_{L Q}^{4}}+D_{2}-\frac{2 D_{4}}{M_{L Q}^{2}}\right)
$$

using unitary gauge. Here $s, t=1-6$ labels the six fermions with the quantum numbers of charged leptons. Note that after summation over the internal leptons the result is finite due to the GIM-like cancellation originating 
from our unitary rotation matrices. The standard loop functions $D_{x} \equiv D_{x}\left(M_{L Q}, M_{L Q}, m_{s}, m_{t}\right)$ are defined as

$$
\begin{aligned}
& \frac{16 \pi^{2}}{i} D_{x}\left(m_{1}, m_{2}, m_{3}, m_{4}\right) \\
& =\int \frac{d^{d} k}{(2 \pi)^{d}} \frac{\left(k^{2}\right)^{x / 2}}{\left(k^{2}-m_{1}^{2}\right)\left(k^{2}-m_{2}^{2}\right)\left(k^{2}-m_{3}^{2}\right)\left(k^{2}-m_{4}^{2}\right)}
\end{aligned}
$$

\section{PHENOMENOLOGICAL ANALYSIS}

Let us first consider $R(D)$ and $R\left(D^{*}\right)$ where the least number of free parameters enters. In order to get a sizable effect, the mixing of $L_{3}^{L}$ with the tau lepton $\ell_{3}^{L}$ should be large. Assuming it to be maximal (i.e., $M_{33}^{L}=m_{33}^{L}$ ), we show the regions preferred by $R\left(D^{(*)}\right)$ in the left plot of Fig. 2 for $M_{L Q}=2 \mathrm{TeV}$. From this we can see that also the mixing between $Q_{3}^{L}$ and $q_{3}^{L}\left(s_{3}^{Q}\right)$, as well as the misalignment between the quark and lepton Yukawa couplings of the second and third generations $\left(s_{23}^{q \ell}\right)$ should be sizable. Our model predicts a significant enhancement of $B_{s} \rightarrow$ $\tau^{+} \tau^{-}[25,28,59,60]$ compared to the SM prediction since this process is in our setup mediated at tree-level with order one couplings.

Let us now turn to the explanation of $b \rightarrow s \ell^{+} \ell^{-}$data. Assuming the absence of mixing with leptons of the first generation, we are safe from processes like $\mu \rightarrow e \gamma$ or $b \rightarrow$ sue [47] and get the right effect in $R(K)$ and $R\left(K^{*}\right)$. Assuming maximal mixing for the third generation quarks and leptons, we show the preferred region from $b \rightarrow s \ell^{+} \ell^{-}$ in the right plot of Fig. 2. This region overlaps with the one from $R\left(D^{(*)}\right)$ for small mixing between the second generation fermions $\left(s_{2}^{Q, L}\right)$ where the predicted branching ratio for $B \rightarrow K \tau \mu$ is automatically compatible with the experimental bounds. However, the predicted rate is still sizable and well within the reach of future measurements.

So far, we did not specify the absolute mass scale of the vectorlike fermions since it did not enter any of the observables. However, for $B_{s}-\bar{B}_{s}$ mixing, the masses of the vectorlike leptons are crucial. In fact, since we calculated $B_{s}-\bar{B}_{s}$ mixing in unitary gauge, the effects of Goldstone bosons are automatically included and, therefore, the result scales proportional to $\left(M^{L}\right)^{2}$ (like the SM contribution is proportional to $m_{t}^{2}$ ). Thus, in order to respect the $B_{s}-\bar{B}_{s}$ mixing bounds while still accounting for $R\left(D^{(*)}\right)$, rather light vectorlike leptons are required. We checked that the $B_{s}-\bar{B}_{s}$ mixing bounds are respected for masses around $500 \mathrm{GeV}$. Since these are third generation leptons, this is compatible with the bounds from direct LHC searches [61,62]. Anyway, since we only included the effect of the Goldstone bosons and not of physical Higgses in this calculation, this should only be understood as a proof that $B_{s}-\bar{B}_{s}$ mixing does not rule out large effects in $R\left(D^{(*)}\right)$. A more precise prediction would require to specify the Higgs sector explicitly and would be, therefore, subject to more model dependence.

\section{INCLUDING THE EW SYMMETRY BREAKING}

Finally let us outline a possible UV completion of the Higgs sector which can lead to the desired EW symmetry breaking extending the previously considered particle content to that shown in Table II. Here we introduced two approximate global symmetries $U(1)_{L, Q}$ and four Higgs fields $\Sigma_{L_{a}}$ and $\Sigma_{Q_{a}}, a=1,2$, which generate the vectorlike masses for $\left(Q_{L}, Q_{R}^{\prime}\right),\left(L_{L}, L_{R}^{\prime}\right),\left(Q_{R}, Q_{L}^{\prime}\right)$, and $\left(L_{R}, L_{L}^{\prime}\right)$ as in Sec. II. $\Phi_{q}$ and $\Phi_{\ell}$ will generate the SM
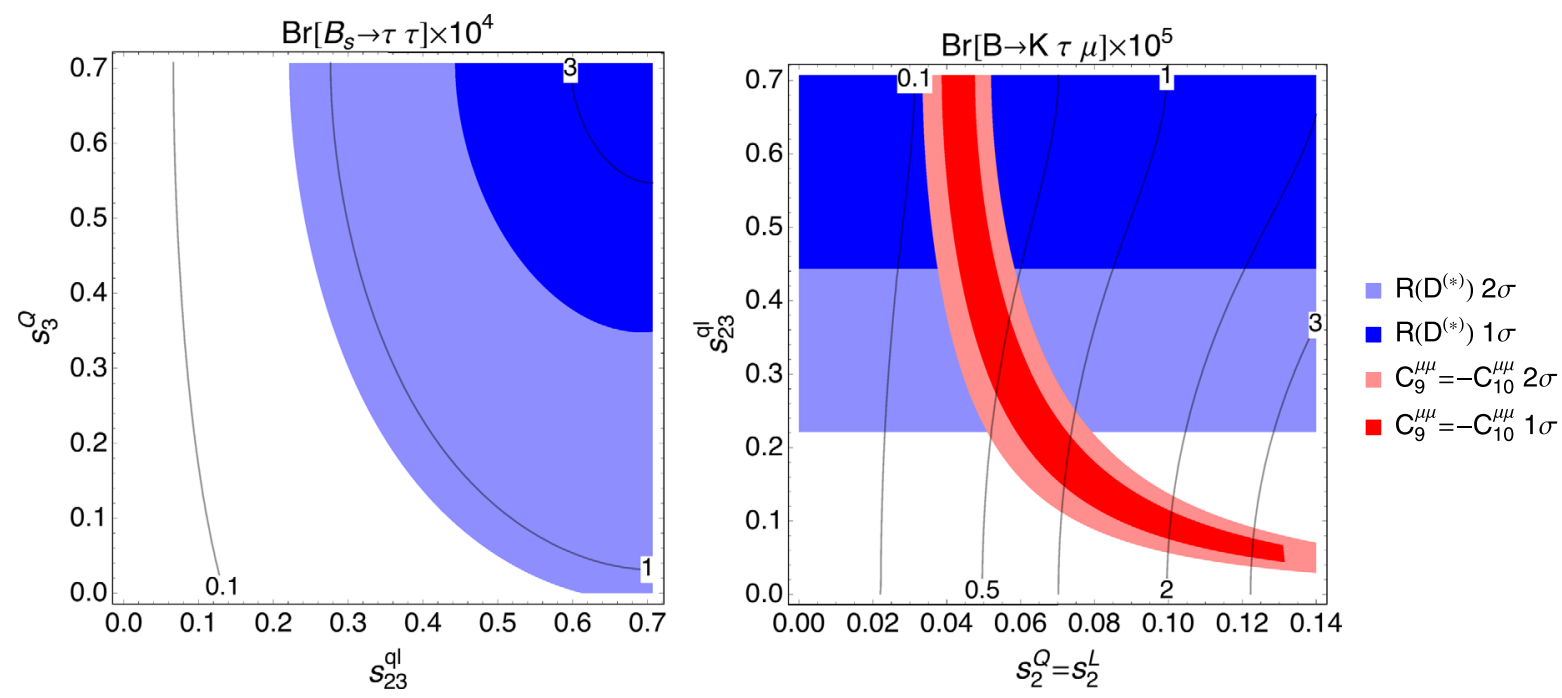

FIG. 2. Left: Allowed regions from $R\left(D^{(*)}\right)$ for $M_{L Q}=2 \mathrm{TeV}$ and $s_{3}^{L}=1 / \sqrt{2}$. Here we used the weighted average for $R(D)$ and $R\left(D^{*}\right)$. The contour lines denote $\operatorname{Br}\left(B_{s} \rightarrow \tau^{+} \tau^{-}\right) \times 10^{4}$. Right: Combined results for $R\left(D^{(*)}\right)$ and $b \rightarrow s \ell^{+} \ell^{-}$, and contours for $\left[\operatorname{Br}\left(B \rightarrow K \tau^{+} \mu^{-}\right)+\operatorname{Br}\left(B \rightarrow K \tau^{-} \mu^{+}\right)\right] / 2$. The red region is preferred by the global fit to $b \rightarrow s \ell^{+} \ell^{-}$data. 
fermion masses and mixing. $\Phi$ will break the $S U(4) \times$ $S U(2)_{L} \times S U(2)_{R}$ symmetry down to the SM gauge symmetry and gives Majorana masses to the right-handed neutrinos. We choose the vevs for $\Sigma_{L_{a}}, \Sigma_{Q_{a}}, a=1,2, \Phi_{q}$, and $\Phi_{\ell}$ as follows

$$
\begin{aligned}
& \Sigma_{L_{a}}=\operatorname{diag}\left(0,0,0, v_{L_{a}}\right), \quad \Sigma_{Q_{a}}=\operatorname{diag}\left(v_{Q_{a}}, v_{Q_{a}}, v_{Q_{a}}, 0\right), \\
& \Phi_{q}=\operatorname{diag}\left(v_{d}, v_{u}\right), \quad \Phi_{\ell}=\operatorname{diag}\left(v_{\ell}, v_{\nu}\right) .
\end{aligned}
$$

The Lagrangian for vectorlike particle masses and SM fermion masses are

$$
\begin{aligned}
-\mathcal{L}= & y_{i j}^{L_{a}} \bar{X}_{i}^{L} Y_{j}^{R} \Sigma_{L_{a}}+y_{i j}^{Q_{a}} \bar{Y}_{i}^{L} Y_{j}^{R} \Sigma_{Q_{a}}+y_{i j}^{L_{a}^{\prime}} \bar{X}_{i}^{R} Z_{j}^{L} \Sigma_{L_{a}} \\
& +y_{i j}^{Q_{a}^{\prime}} \bar{Z}_{i}^{R} Z_{j}^{L} \Sigma_{Q_{a}}+y_{i j}^{q} \bar{X}_{i}^{L} X_{j}^{R} \Phi_{q}+y_{i j}^{\ell} \bar{Y}_{i}^{L} Z_{j}^{R} \Phi_{\ell} \\
& +y_{i j}^{N} \bar{Z}_{i}^{R} Z_{j}^{R} \Phi+\text { H.c. }
\end{aligned}
$$

Thus, without further contributions, the up-type quark mass matrix would be proportional to the down-type quark mass matrix. To generate the couplings between the SM lefthanded fermions and massive gauge bosons and solve the quark mass problem, we introduce the following terms which violate the $U(1)_{Q} \times U(1)_{L}$ global symmetries

$$
\begin{aligned}
-\mathcal{L}= & h_{i j}^{Q} \bar{X}_{i}^{L} Y_{j}^{R} \Sigma_{Q}+h_{i j}^{L} \bar{Y}_{i}^{L} Y_{j}^{R} \Sigma_{L}+h_{i j}^{q} \bar{X}_{i}^{L} X_{j}^{R} \Phi_{\ell} \\
& +h_{i j}^{\ell} \bar{Y}_{i}^{L} Z_{j}^{R} \Phi_{q}+\text { H.c. },
\end{aligned}
$$

where we expect the above Yukawa couplings to be relatively small compared to these in Eq. (25) due to $U(1)_{Q} \times U(1)_{L}$ global symmetry breaking. Notice that the first line gives rise to mass terms of the form given in Eq. (1). We defer to future publication a complete analysis of this and other possible completions of our setup, including the flavor effects induced by the extra Higgses.

\section{CONCLUSIONS AND OUTLOOK}

In this article, we presented a renormalizable phenomenologically valid $\mathrm{TeV}$ scale model of a vector leptoquarks with flavor dependent couplings. The model is an extension of the PS model obtained by adding three generations of vectorlike fermions which are in fundamental representations of $S U(4)$. Our model can successfully address the observed deviations from the SM predictions in semileptonic $B$ decays $\left(R\left(D^{(*)}\right)\right.$ as well as in $b \rightarrow s \ell^{+} \ell^{-}$ transitions) and easily account for the anomaly in the anomalous magnetic moment of the muon too, as we comment below. An explanation of $R\left(D^{(*)}\right)$ predicts a significant enhancement of $B_{s} \rightarrow \tau^{+} \tau^{-}$and once also $b \rightarrow$ $s \ell^{+} \ell^{-}$is included, sizable rates for $b \rightarrow \tau \mu$ processes must occur. Also bounds from $B_{s}-\bar{B}_{s}$ mixing are respected for not too heavy vectorlike leptons.

The longstanding anomaly in the anomalous magnetic moment of the muon (at the $3 \sigma$ level) might also be related to the $B$-physics anomalies [17,19,25,35,63-68]. Our model can in principle explain this discrepancy, as long as one introduces couplings of heavy down-type quarks to the SM Higgs doublet. Following Eq. (10), we call the vectorlike fermions with the same quantum numbers as right-handed down-quarks $D_{i}^{R}$ and $D_{i}^{\prime L}$. Then the coupling to the Higgs is given by $\lambda_{i i} \bar{D}_{i}^{\prime L} \phi D_{i}^{R}$ where our coupling of interest is $\lambda_{22}$. For vectorlike quarks and leptoquark of equal mass $M$, the resulting contribution is $\delta a_{\mu} \approx \frac{m_{\mu}}{4 \pi^{2}} \frac{2 v \lambda_{22}}{M^{2}}$. Since $\lambda_{22}$ does not enter in other observables discussed to far, it can simply be adjusted to account for the anomaly. Note that this is possible for natural values (smaller than 0.1 ).

\section{ACKNOWLEDGMENTS}

The work of A.C. is supported by an Ambizione Grant of the Swiss National Science Foundation (PZ00P2_154834). This research was supported in part by the Projects No. 11475238 and No. 11647601 supported by the National Natural Science Foundation of China, and by the Key Research Program of Frontier Science, CAS. A. C. thanks Dario Müller for useful comments on the manuscript. We are especially grateful to Luca Di Luzio, Admir Greljo, and Marco Nardecchia for discussions and for pointing out that, in an earlier version of the paper, lepton and quark rotations were not independent, such that the desired pattern for the masses could not be achieved.

Note added.-During the completion of the article, a model of vector leptoquarks also based on the PS group was presented in Ref. [69] (for an earlier attempt see also Ref. [70]). While in our model all fermions transform in the fundamental of $S U(4)$, in Ref. [69] the gauge group contains another $S U(3)$ factor and the SM-like fermions are singlets of $S U(4)$. Also note that in our model only the leptoquark has flavor nonuniversal couplings while in the model of Ref. [69] also the heavy $Z^{\prime}$ and the heavy gluons in general acquire flavor violating couplings. 
[1] B. Capdevila, A. Crivellin, S. Descotes-Genon, J. Matias, and J. Virto, J. High Energy Phys. 01 (2018) 093.

[2] W. Altmannshofer, P. Stangl, and D. M. Straub, Phys. Rev. D 96, 055008 (2017).

[3] G. D’Amico, M. Nardecchia, P. Panci, F. Sannino, A. Strumia, R. Torre, and A. Urbano, J. High Energy Phys. 09 (2017) 010.

[4] L.-S. Geng, B. Grinstein, S. Jager, J. Martin Camalich, X.-L. Ren, and R.-X. Shi, Phys. Rev. D 96, 093006 (2017).

[5] M. Ciuchini, A. M. Coutinho, M. Fedele, E. Franco, A. Paul, L. Silvestrini, and M. Valli, Eur. Phys. J. C 77, 688 (2017).

[6] G. Hiller and I. Nisandzic, Phys. Rev. D 96, 035003 (2017).

[7] A. K. Alok, B. Bhattacharya, A. Datta, D. Kumar, J. Kumar, and D. London, Phys. Rev. D 96, 095009 (2017).

[8] T. Hurth, F. Mahmoudi, D. Martinez Santos, and S. Neshatpour, Phys. Rev. D 96, 095034 (2017).

[9] Y. Amhis et al., Eur. Phys. J. C 77, 895 (2017).

[10] R. Aaij et al. (LHCb Collaboration), Phys. Rev. Lett. 113, 151601 (2014).

[11] R. Aaij et al. (LHCb Collaboration), J. High Energy Phys. 08 (2017) 055.

[12] B. Bhattacharya, A. Datta, D. London, and S. Shivashankara, Phys. Lett. B 742, 370 (2015).

[13] L. Calibbi, A. Crivellin, and T. Ota, Phys. Rev. Lett. 115, 181801 (2015).

[14] S. Fajfer and N. Košnik, Phys. Lett. B 755, 270 (2016).

[15] A. Greljo, G. Isidori, and D. Marzocca, J. High Energy Phys. 07 (2015) 142.

[16] R. Barbieri, G. Isidori, A. Pattori, and F. Senia, Eur. Phys. J. C 76, 67 (2016).

[17] M. Bauer and M. Neubert, Phys. Rev. Lett. 116, 141802 (2016).

[18] S. M. Boucenna, A. Celis, J. Fuentes-Martin, A. Vicente, and J. Virto, J. High Energy Phys. 12 (2016) 059.

[19] D. Das, C. Hati, G. Kumar, and N. Mahajan, Phys. Rev. D 94, 055034 (2016).

[20] D. Bečirević, S. Fajfer, N. Košnik, and O. Sumensari, Phys. Rev. D 94, 115021 (2016).

[21] S. Sahoo, R. Mohanta, and A. K. Giri, Phys. Rev. D 95, 035027 (2017).

[22] B. Bhattacharya, A. Datta, J.-P. Guevin, D. London, and R. Watanabe, J. High Energy Phys. 01 (2017) 015.

[23] R. Barbieri, C. W. Murphy, and F. Senia, Eur. Phys. J. C 77, 8 (2017).

[24] A. K. Alok, D. Kumar, J. Kumar, and R. Sharma, arXiv: 1704.07347.

[25] A. Crivellin, D. Mueller, and T. Ota, J. High Energy Phys. 09 (2017) 040.

[26] C.-H. Chen, T. Nomura, and H. Okada, Phys. Lett. B 774, 456 (2017).

[27] I. Doršner, S. Fajfer, D. A. Faroughy, and N. Košnik, J. High Energy Phys. 10 (2017) 188.

[28] D. Buttazzo, A. Greljo, G. Isidori, and D. Marzocca, J. High Energy Phys. 11 (2017) 044.

[29] D. A. Faroughy, A. Greljo, and J. Kamenik, Phys. Lett. B 764, 126 (2017).

[30] F. Feruglio, P. Paradisi, and A. Pattori, Phys. Rev. Lett. 118, 011801 (2017).
[31] A. Crivellin, C. Greub, and A. Kokulu, Phys. Rev. D 86, 054014 (2012).

[32] M. Tanaka and R. Watanabe, Phys. Rev. D 87, 034028 (2013).

[33] A. Celis, M. Jung, X.-Q. Li, and A. Pich, J. High Energy Phys. 01 (2013) 054.

[34] A. Crivellin, A. Kokulu, and C. Greub, Phys. Rev. D 87, 094031 (2013).

[35] A. Crivellin, J. Heeck, and P. Stoffer, Phys. Rev. Lett. 116, 081801 (2016).

[36] C.-H. Chen and T. Nomura, Eur. Phys. J. C 77, 631 (2017).

[37] M. Freytsis, Z. Ligeti, and J. T. Ruderman, Phys. Rev. D 92, 054018 (2015).

[38] A. Celis, M. Jung, X.-Q. Li, and A. Pich, Phys. Lett. B 771, 168 (2017).

[39] M. A. Ivanov, J. G. Koerner, and C.-T. Tran, Phys. Rev. D 95, 036021 (2017).

[40] X.-Q. Li, Y.-D. Yang, and X. Zhang, J. High Energy Phys. 08 (2016) 054.

[41] R. Alonso, B. Grinstein, and J. Martin Camalich, Phys. Rev. Lett. 118, 081802 (2017).

[42] A. G. Akeroyd and C.-H. Chen, Phys. Rev. D 96, 075011 (2017).

[43] P. Q. Hung, A. J. Buras, and J. D. Bjorken, Phys. Rev. D 25, 805 (1982).

[44] G. Valencia and S. Willenbrock, Phys. Rev. D 50, 6843 (1994).

[45] J. C. Pati and A. Salam, Phys. Rev. D 10, 275 (1974); 11, 703(E) (1975).

[46] A. Crivellin, C. Greub, F. Saturnino, and D. Müller, arXiv: 1807.02068.

[47] A. Crivellin, D. Mueller, A. Signer, and Y. Ulrich, Phys. Rev. D 97, 015019 (2018).

[48] M. Aaboud et al. (ATLAS Collaboration), J. High Energy Phys. 10 (2017) 182.

[49] A. Crivellin, G. D'Ambrosio, M. Hoferichter, and L. C. Tunstall, Phys. Rev. D 93, 074038 (2016).

[50] S. Fajfer, J. F. Kamenik, and I. Nisandzic, Phys. Rev. D 85, 094025 (2012).

[51] H. Na, C. M. Bouchard, G. P. Lepage, C. Monahan, and J. Shigemitsu (HPQCD Collaboration), Phys. Rev. D 92, 054510 (2015); 93, 119906(E) (2016).

[52] A. Crivellin, L. Hofer, J. Matias, U. Nierste, S. Pokorski et al., arXiv:1504.07928.

[53] J. P. Lees et al. (BABAR Collaboration), Phys. Rev. D 86, 012004 (2012).

[54] C. Bobeth, M. Gorbahn, T. Hermann, M. Misiak, E. Stamou, and M. Steinhauser, Phys. Rev. Lett. 112, 101801 (2014).

[55] C. Bobeth, in Proceedings of the 49th Rencontres de Moriond on Electroweak Interactions and Unified Theories, La Thuile, Italy, 2014 (2014), pp. 75-80.

[56] C. Bobeth, M. Misiak, and J. Urban, Nucl. Phys. B574, 291 (2000).

[57] T. Huber, E. Lunghi, M. Misiak, and D. Wyler, Nucl. Phys. B740, 105 (2006).

[58] R. Aaij et al. (LHCb Collaboration), Phys. Rev. Lett. 118, 251802 (2017).

[59] R. Alonso, B. Grinstein, and J. M. Camalich, J. High Energy Phys. 10 (2015) 184. 
[60] D. Choudhury, A. Kundu, R. Mandal, and R. Sinha, Phys. Rev. Lett. 119, 151801 (2017).

[61] A. Falkowski, D. M. Straub, and A. Vicente, J. High Energy Phys. 05 (2014) 092.

[62] R. Dermisek, J. P. Hall, E. Lunghi, and S. Shin, J. High Energy Phys. 12 (2014) 013.

[63] G. Belanger, C. Delaunay, and S. Westhoff, Phys. Rev. D 92 , 055021 (2015).

[64] W. Altmannshofer, M. Carena, and A. Crivellin, Phys. Rev. Lett. (2016); Phys. Rev. D 94, 095026 (2016).

[65] C.-H. Chen, T. Nomura, and H. Okada, Phys. Rev. D 94, 115005 (2016).
[66] P. Arnan, L. Hofer, F. Mescia, and A. Crivellin, J. High Energy Phys. 04 (2017) 043.

[67] E. Coluccio Leskow, A. Crivellin, G. D’Ambrosio, and D. Mueller, Phys. Rev. D 95, 055018 (2017).

[68] S. Di Chiara, A. Fowlie, S. Fraser, C. Marzo, L. Marzola, M. Raidal, and C. Spethmann, Nucl. Phys. B923, 245 (2017).

[69] L. Di Luzio, A. Greljo, and M. Nardecchia, Phys. Rev. D 96, 115011 (2017).

[70] N. Assad, B. Fornal, and B. Grinstein, Phys. Lett. B 777, 324 (2018). 